\title{
Trisporic Acid Synthesis in Homothallic and Heterothallic Mucorales
}

\author{
By BERTINE A. WERKMAN AND H. VAN DEN ENDE \\ Department of Plant Physiology, University of Amsterdam, Amsterdam, \\ The Netherlands
}

(Received 7 January 1974)

\begin{abstract}
SUMMAR Y
The synthesis of trisporic acids, the sexual hormones, proceeds in a similar way in mated cultures of the heterothallic fungi Mucor mucedo and Blakeslea trispora. In both species precursors are secreted by both mating types and these can be converted to trisporic acids by the sexual partner. Precursor production is strongly stimulated by trisporic acids. In homothallic species the presence of biologically active substances can be demonstrated, which have characteristics similar to those found in heterothallic strains. Moreover, precursors of trisporic acids isolated from Mucor mucedo are readily converted to trisporic acids by homothallic species. These results suggest a close relationship between the hormonal systems of homothallic and heterothallic species.
\end{abstract}

\section{INTRODUCTION}

Trisporic acids, substances which induce the formation of sexual structures (zygophores) in Mucor mucedo, are produced by mated cultures of $M$. mucedo, Blakeslea trispora and Phycomyces blakesleeanus in the order Mucorales (Gooday, 1973). In B. trispora there is evidence that each mating type produces specific precursors which are converted to trisporic acids by the opposite mating type (Sutter, Capage, Harrison \& Keen, I973). Trisporic acids have a stimulatory effect on precursor production in both mating types (Werkman \& van den Ende, 1973), which may help to explain the relatively large yields of trisporic acids in mated cultures of $B$. trispora. We wondered whether the same mechanism of trisporic acid formation operates in other heterothallic Mucors which produce much lower amounts of trisporic acids. Here $M$. mucedo has been studied as: (i) it has often been used in sexual studies in the Mucorales; (ii) it produces only small amounts of trisporic acids in mated cultures (e.g. Gooday, 1973); and (iii) it is not closely allied to B. trispora in a morphological sense.

We were also interested in the hormonal aspects of sexuality in related homothallic species. The occurrence of interspecific sexual reactions between homothallic and heterothallic strains suggests similar sexual mechanisms. If trisporic acids or related substances are involved in zygophore formation in homothallic species, one might suppose that in these species at least part of the biosynthetic pathway to trisporic acids would be operative. Therefore we investigated whether homothallic strains do produce biologically active substances similar to those isolated from heterothallic strains and whether they can convert precursor material derived from heterothallic strains to trisporic acids. 


\section{METHODS}

Cultures. The strains used in the present investigation were: Mucor mucedo Bref. strain Saito (minus), CBSI09. 16; Mucor mucedo Bref. strain Burgeff (plus), CBSI44.24; Mucor genevensis Lendner, NRRLI755; Zygorhynchus moelleri Vuill., CBS501.66; Z. heterogamus Vuill., CBS405.58; and Syzygites megalocarpus Ehrenb. ex Fr., CBS514.66. All strains were obtained from the Centraal Bureau voor Schimmelcultures, Baarn, The Netherlands, except M. genevensis which was kindly provided by Dr J. J. Ellis, U.S. Department of Agriculture, Peoria, Illinois, U.S.A.

All strains were cultivated at $20{ }^{\circ} \mathrm{C}$ in the dark in $30 \%(\mathrm{v} / \mathrm{v})$ beer wort adjusted to $\mathrm{pH} 8$ before sterilization. Submerged cultures were grown for 4 days in $100 \mathrm{ml}$ portions in $300 \mathrm{ml}$ Erlenmeyer flasks on a reciprocal shaker; surface cultures were grown for an equal period of time in $30 \mathrm{ml}$ portions in Petri dishes.

Extraction and incubation of biologically active substances was carried out as described previously (Werkman \& van den Ende, I973), except that the hexane extraction was omitted. One unit of zygophore-inducing material is defined as the smallest amount which induces the formation of zygophores in Mucor mucedo at $20^{\circ} \mathrm{C}$ in the dark (van den Ende \& Stegwee, 197I). The total amounts were estimated by serial dilutions (expressed in units). All other procedures were as described earlier (van den Ende, Werkman \& van den Briel, 1972). Trisporic acids were derived from Blakeslea trispora and isolated as described earlier (van den Ende et al. 1972).

\section{RESULTS}

Production of precursors and conversion to trisporic acids in Mucor mucedo. The following results demonstrate that the liquid from unmated $M$. mucedo cultures contains neutral substances which are converted to trisporic acids by the sexual partner. Radioactive plus mycelia (mycelia grown in culture media containing [U-14 C]glucose) were incubated in a solution of glucose and trisporic acids to stimulate precursor production (see below). From the incubation liquid, neutral, ether-soluble materials were extracted and purified. Samples were assayed for biological activity towards $M$. mucedo-minus. Substances that gave a positive reaction were incubated with $M$. mucedo-minus mycelium in a liquid medium. After 2 days the incubation liquids were tested for the presence of trisporic acids by extraction and DEAE Sephadex chromatography (Werkman \& van den Ende, 1973). By the same procedure radioactive precursor material was isolated from radioactive $M$. mucedo-minus cultures and incubated with $M$. mucedo-plus cultures, after which trisporic acids were isolated and purified by fractionation on columns of DEAE Sephadex. The radioactivity, biological activity and u.v.-absorption profiles are presented in Fig. I $a \mathrm{i}, b \mathrm{i}$. Part of the radioactive neutral compounds from both plus and minus (Fig. I $a \mathrm{i}$ and $b \mathrm{i}$, respectively) had been converted by the opposite mating partner to radioactive compounds that were retained on DEAE Sephadex. These compounds were eluted at the same ionic strength as trisporic acids, were biologically active on both plus and minus $M$. mucedo and exhibited the same u.v.-absorption spectrum as trisporic acids; we therefore assume that they were trisporic acids.

When the radioactive neutral precursors were incubated alone or with non-radioactive cultures of the same mating type, they were unstable, as much of the radioactivity was lost within 2 days. Trisporic acids were not formed.

In a further experiment, ten Petri dishes, containing $20 \mathrm{ml}$ culture medium with or without $54 \mu \mathrm{g}$ trisporic acids, were inoculated with plus or minus Mucor mucedo. After four days 
the media were collected, adjusted to $\mathrm{pH} 8$ and extracted exhaustively with ether. The ether fraction was washed with $5 \%(w / v)$ sodium bicarbonate and water, and the ether evaporated in vacuo. The residues were taken up in $5 \mathrm{ml}$ water; with a series of dilutions ( $\mathrm{I}: 3$ ) the dilution was determined which still induced zygophores in the standard assay with $M$. mucedo ( $0.5 \mathrm{ml}$ per application) of opposite mating type. Trisporic acids stimulated up to tenfold the synthesis of biologically active precursors in both plus and minus $M$. mucedo (Table 1 ).

Isolation of zygophore-inducing substances from homothallic strains. The culture media from Mucor genevensis, Zygorhynchus moelleri, Z. heterogamus and Syzygites megalocarpus (fresh weights approx. $50 \mathrm{~g}$ ) were extracted with ether, and the extracts subjected to DEAE Sephadex column chromatography as described above. Fractions were assayed for zygophore-inducing capacity towards $M$. mucedo-plus and $M$. mucedo-minus. In extracts of culture media from $Z$. moelleri and $Z$. heterogamus small amounts of acidic material were present with the same elution pattern on DEAE Sephadex as trisporic acids and with zygophore-inducing capacity towards both plus and minus mating type of $M$. mucedo. In extracts of $Z$. moelleri culture media (but not of $Z$. heterogamus) neutral material was present with the same chromatographic characteristics and biological activity as trisporic acidprecursor material from $M$. mucedo-minus. From the other homothallic strains no biologically active substances could be detected. These results suggest that at least in $Z$. moelleri and $Z$. heterogamus sexual morphogenesis is governed by similar low molecular weight substances as in heterothallic systems.

Conversion of precursors from Mucor mucedo to trisporic acids by homothallic strains. Radioactive trisporic acid-precursor materials isolated from $M$. mucedo-plus and -minus as described above were incubated with submerged cultures of $M$. genevensis, Zygorhynchus moelleri, Z. heterogamus and Syzygites megalocarpus. After 2 days the media were extracted after acidification with ether. The extracts were subjected to DEAE Sephadex column chromatography and the eluates were assayed for biological activity and radioactivity. All four strains appeared able to convert precursor material obtained from $M$. mucedo-plus to trisporic acids (Fig. I $a$ ii to v), since those fractions from the column that would contain authentic trisporic acids contained zygophore-inducing activity towards both mating types of $M$. mucedo. In controls of extracts of corresponding cultures incubated without precursor material, or the neutral precursor material incubated alone, no biological activity was found in the fractions in which trisporic acids might have been present. (This is not opposed to the previous isolation of zygophore-inducing substances from homothallic cultures, since in this experiment the total fresh weight of the cultures used was 14 times less.) In all cultures except $Z$. heterogamus radioactivity was associated with the biologically active fractions. Taken together with a measurable u.v.-absorption spectrum similar to that of trisporic acids, this suggests that in $M$. genevensis, $Z$. moelleri and $S$. megalocarpus the neutral precursor material of trisporic acids derived from $M$. mucedo-plus is as easily converted to trisporic acids as in $M$. mucedo-minus. In $Z$. heterogamus detectable trisporic acids were formed from added plus precursors, but considerably less efficiently than in other homothallic fungi. However, $Z$. heterogamus was superior to the other homothallic fungi in converting $M$. mucedo-minus neutral precursors to trisporic acids, although not as efficient as $M$. mucedo-plus (Fig. I bii to v). The other homothallic strains, with the possible exception of $S$. megalocarpus from which some biological activity was recovered with the same retention as trisporic acids, showed no detectable conversion of minus neutral precursors to trisporic acids (Fig. I $b$ ii to v). After incubation of $M$. genevensis and $Z$. moelleri with the plus precursors, no starting material could be recovered, but from all the other incubations some unchanged starting material could be detected by bioassays. 
(a) Extracts from M. mucedo-pluts
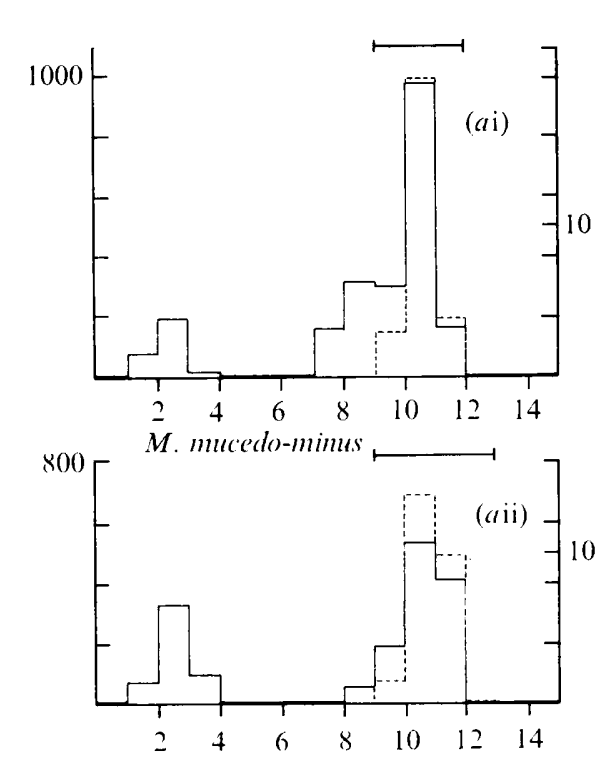

(b) Extracts from $M$. mucedo-minus
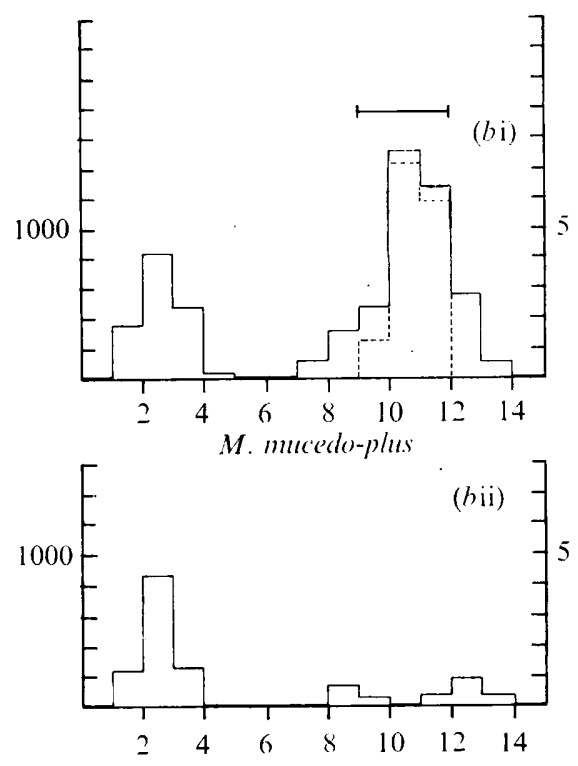
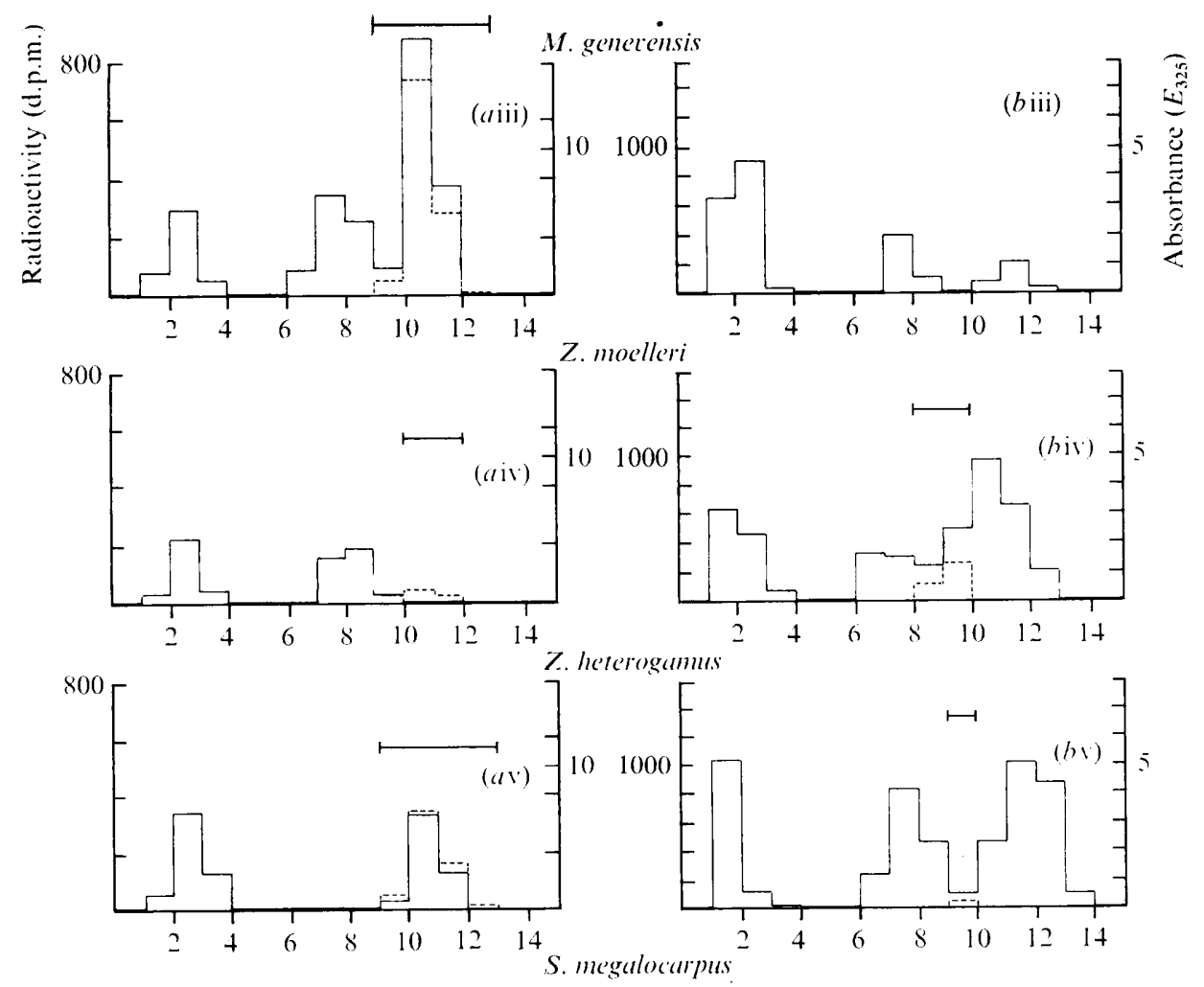

Fraction numbers from DEAE Sephadex elution

Fig. I. For legend see facing page. 


\section{Table I. Influence of trisporic acids on neutral precursor production in plus and minus mycelium of $M$. mucedo}

The total amounts determined for plus and for minus precursor materials are not comparable. Values are averages of duplicates. Deviations from the average are indicated.

$\begin{array}{lcc}\begin{array}{c}\text { Mating } \\ \text { type }\end{array} & \begin{array}{c}\text { Trisporic acids } \\ \text { added }\end{array} & \begin{array}{c}\text { Neutral precursor } \\ \text { material produced } \\ \text { (units) }\end{array} \\ \text { plus } & \text { No } & 69 \pm 39 \\ \text { plus } & \text { Yes } & 891 \pm 81 \\ \text { minus } & \text { No } & 207 \pm 117 \\ \text { minus } & \text { Yes } & 1863 \pm 1053\end{array}$

\section{DISCUSSION}

Blakeslea trispora was the fungus first shown to produce trisporic acids and the fungus used to investigate how hormone synthesis is initiated (Caglioti et al. 1964, Sutter et al. 1973). As Gooday (1973) remarked, however, it should be remembered that this species may not necessarily exhibit a situation representative for all heterothallic Mucorales, because it gives excessively high hormone yields. Therefore any scheme proposed for the sexual behaviour of B. trispora should be tested with Mucor mucedo and other species. Our results show that the hormonal situation in these two fungi may be very similar.

Part of this study was concerned with homothallic Mucorales, because the suggestion has repeatedly been made (e.g. Gooday, 1973) that in these species sexuality is also controlled by trisporic acids. This suggestion was based on the following observations: (i) interspecific though incomplete sexual reactions between homo- and heterothallic species are very common (Burgeff, 1924; Satina \& Blakeslee, 1930); (ii) zygospore formation in seemingly sterile homothallic strains can be induced by contact with other homo- or heterothallic species (Schipper, I97I); (iii) incubation of the plus strain of B. trispora or M. mucedo

Fig. I. Radioactivities and biological activities after DEAE Sephadex column chromatography of extracts of cultures incubated with trisporic acid-precursor material. (ai) Twenty-eight submerged plus $M$. mucedo cultures were grown in the presence of $30 \mu \mathrm{Ci}\left[\mathrm{U}-{ }^{14} \mathrm{C}\right]$ glucose $(120 \mathrm{nmol})$. After harvesting, the mycelia (approx. $5 \mathrm{~g}$ fresh weight each) were incubated in $100 \mathrm{ml}$ of a solution of $5 \mu \mathrm{g}$ trisporic acids (isolated as described previously, van den Ende et al. 1972) per $\mathrm{ml}$ and $1 \%$ (w/v) glucose for 4 days (with one further addition of trisporic acid and glucose). The collected incubation liquids were extracted with diethyl ether after adjusting the $\mathrm{pH}$ to 8. After evaporating the ether from the extract in vacuo, the aqueous residue was put on a DEAE Sephadex column which was eluted with 0.01 ionic strength tris- $\mathrm{HCl}$ buffer, $\mathrm{pH} 8.5$. The fractions with biological activity towards $M$. mucedo-minus were pooled and one-seventh added to $M$. mucedo-minus cultures (total fresh weight, $3.5 \mathrm{~g}$; added radioactivity, $30518 \mathrm{~d} . \mathrm{p} . \mathrm{m}$.). After $48 \mathrm{~h}$ the culture liquids were acidified to $\mathrm{pH} 2$ and extracted with ether to yield any trisporic acids. From the acid ether fraction, the solvent was evaporated in vacuo, and the aqueous residue subjected to DEAE Sephadex chromatography, with a discontinuous gradient of tris- $\mathrm{HCl}$ buffer $\mathrm{pH} 8 \cdot 5$. containing an increasing concentration of $\mathrm{KCl}$ (starting at total ionic strength of 0.01 , van den Ende et al. 1972). Fractions $(5 \mathrm{ml})$ were collected, in which radioactivities and biological activities towards both plus and minus strains of $M$. mucedo were determined. (bi) A slightly modified procedure was followed to obtain radioactive material from $M$. mucedo-minus cultures. Twenty-eight unlabelled minus mycelia were incubated in $100 \mathrm{ml}$ of a solution containing $\mathrm{IO} \mu \mathrm{Ci}\left[\mathrm{U}-{ }^{14} \mathrm{C}\right]$ glucose $(40 \mathrm{nmol}), 5 \mu \mathrm{g}$ trisporic acids/ $\mathrm{ml}$ and $2 \%(\mathrm{w} / \mathrm{v})$ glucose for 4 days. The procedure described above was then followed. The radioactive extract was incubated with $M$. mucedo-plus cultures (total fresh weight, $2.3 \mathrm{~g}$; added radioactivity, 175545 d.p.m.). ( $a$ ii to v) Radioactive $M$. mucedo-plus material as in ( $a$ i) added to cultures of $M$. genevensis, $Z$. moelleri, $Z$. heterogamus and $S$. megalocarpus, respectively. (bii to v) Radioactive $M$. mucedo-minus material as in (bi) added to homothallic cultures, with the same quantities as in $(\mathrm{bi}) . \longrightarrow$, Total radioactivity per fraction; - _ - , u.v. absorbance of peak at $325 \mathrm{~nm}$; $\vdash$ zygophore-inducing activity on both plus and minus mating types of $M$. mucedo. 
with the homothallic Zygorhynchus moelleri gives measurable trisporic acid synthesis (van den Ende, 1968).

In interspecific conjugations between homo- and heterothallic species, some homothallic strains react predominantly with plus, others with minus strains. Therefore, Satina \& Blakeslee (1930) attributed a minus 'tendency' to some homothallic strains, and a plus 'tendency' to others, although yet other homothallic species react with both plus and minus strains. Zygorhynchus moelleri is a homothallic strain with a minus tendency. We have found that this fungus converts precursor material derived from Mucor mucedo-plus about as efficiently as $M$. mucedo-minus, and also produces small amounts of precursor material very similar to that isolated from $M$. mucedo-minus. This explains the production of trisporic acids in a mated culture of $Z$. moelleri and a plus strain of $M$. mucedo or Blakeslea trispora, and the more abundant production of zygophores in M. mucedo-plus than in M. mucedo-minus when mated with $Z$. moelleri.Z. heterogamus, on the other hand, exhibits a plus tendency. Although no 'plus-like' trisporic acid-precursor material has been detected in its culture media, this fungus may convert minus precursors to trisporic acids. The weak sexual responses of this strain seem to be reflected by its rather low efficiency of precursor conversion.

Satina \& Blakeslee (1930) found that Mucor genevensis reacted with plus and minus strains. This character was not reflected in our experiments: our strain effectively converted plus precursors but not minus precursors to trisporic acids.

Syzygites megalocarpus has been described as sexually indifferent to heterothallic species (Satina \& Blakeslee, 1930). However, our strain transformed both plus and minus precursors to trisporic acids. We observed that this species was unable to produce zygospores under standard conditions (described above) but did so abundantly when trisporic acids or its precursors isolated from Mucor mucedo were applied.

Although the study of hormonal aspects of sexuality in homothallic species has just begun, the conclusion that the hormonal system in homo- and heterothallic species of the Mucorales is closely related seems to be justified.

\section{REFERENCES}

BuRgEF, H. (1924). Untersuchungen über Sexualität und Parasitismus bei Mucorineen. I. Botanische Abhandlungen 4, I-I35.

Caglioti, L., Cainelli, G.,'Camerino, B., Mandelli, R., Prieto, A., Salvatori, T. \& Selva, A. (I964). Sulla costituzione degli'acidi trisporici. Chimica e l'industria (Milano) 46, 96I-966.

VAN DEN ENDE, H.(1968). Relationship between sexuality and carotene synthesis in Blakeslea trispora. Journal of Bacteriology 96, 1298-1303.

VAN DEN Ende, H. \& STEGWee, D. (1971). Physiology of sex in Mucorales. Botanical Review 37, 22-36.

VAN Den Ende, H., Werkman, B. A. \& VAN DEN Briel, M. L. (1972). Trisporic acid synthesis in mated cultures of the fungus Blakeslea trispora. Archiv fïr Mikrobiologie 86, 175-184.

Gooday, G. W. (1973). Differentiation in the Mucorales. Symposia of the Society for General Microbiology 23, 269-294.

SAtinA, S. \& Blakeslee, A. F. (1930). Imperfect sexual reactions in homothallic and heterothallic Mucors. Botanical Gazette 90, 299-3II.

SCHIPPER, M. A. A. (I97I). Induction of zygospore production in Mucor saximontensis, an agamic strain of Zygorhynchus moelleri. Transactions of the British Mycological Society 56, I57-158.

Sutter, R. P., Capage, D. A., Harrison, T. L. \& Keen, W. A. (1973). Trisporic acid biosynthesis in separate plus and minus cultures of Blakeslea trispora: identification by Mucor assay of two mating type specific components. Journal of Bacteriology ri4, 1074-1090.

Werkman, B. A. \& VAN DEN ENDE, H. (1973). Trisporic acid synthesis in Blakeslea trispora. Archiv für Mikrobiologie 90,_365-374. 